\title{
Flora and Botanic Tourism Potential of Yaralıgöz (Kastamonu) Education and Observation Forest
}

\author{
Mustafa KARAKOSE ${ }^{*^{*}}$, Salih TERZIOGLU² \\ ${ }^{1}$ Giresun University, Espiye Vocational School, Giresun, TURKEY \\ ${ }^{2}$ Karadeniz Technical University, Faculty of Forestry, Department of Forest Engineering, Trabzon, \\ TURKEY \\ *Corresponding Author: mustafa.karakose@giresun.edu.tr
}

Received Date: 05.11.2018

Accepted Date: 26.02.2019

\section{Abstract}

Aim of Study: In this study, it was aimed to identify the flora of Yaraligöz Education and Observation Forest and revealing its potential in terms of botanic tourism.

Area of study: The study area is the Yaralıgöz Education and Observation Forest, which is within the boundaries of four forest planning units (Devrekani, Tezcan, Şeyhşaban and Karacakaya) belonging to the Kastamonu Regional Directorate. Yaralıgöz Education and Observation Forest is located in the transitional zone between the Euxine province of Euro-Siberian and Irano-Turanian floristic areas in terms of plant geography.

Material and Methods: This research is a flora study and the materials of this stridy included plant specimens collected from Yaralıgöz Mountainbetween 2011 and 2012.

Main Results: With this study, 374 vascular plant taxa were identified. Pteridophyta section were represented by 6 taxa, Pinidae sub-class by 7 taxa, and Magnoliidae subclass by 361 taxa. The largest family was Asteraceae (45; $12.1 \%$ ), followed by Lamiaceae (35; 9.3\%), Rosaceae and Fabaceae (27; 7.2\%). The phytogeographic regions of 190 taxa represented in the study area are as follows: Euro-Siberian 144 (38.7\%), Irano-Turanian $24(6.4 \%)$ and Mediterranean $22(5.9 \%)$. Raunkiaer's life forms showed that Hemicryptophytes with $50.7 \%$ Phanerophytes with $15.7 \%$ and Cryptophytes with $14.9 \%$ were the most frequent life forms.

Highlights: Twenty-three endemic and one rare plant taxa were identified. In addition, new distribution areas were determined for the endemic taxon Acer hyrcanum subsp. keckianum and European spruce. Because of the presence of many characteristic plant species, Yaralıgöz Education and Observation Forest has been identified to have an important botanic tourism potential.

Keywords: Ecotourism, flora, endemic, plant biodiversity, Turkey, Yaralıgöz Mountain.

\section{Yaralıgöz (Kastamonu) Eğitim ve Gözlem Ormanı'nın Florası ve}

\section{Botanik Turizm Potansiyeli}

Öz

Çalışmanın amacı: Bu çalışmada, Yaralıgöz Eğitim ve Gözlem Ormanı'nın florasının belirlenmesi ve botanik turizm açısından potansiyelinin ortaya çıkarılması amaçlanmıştır.

Çalışma alanı: Çalışma alanı Kastamonu Bölge Müdürlüğü'ne ait dört orman planlama birimi (Devrekani, Tezcan, Şeyhşaban ve Karacakaya) sınırları içerisinde kalan Yaralıgöz Eğitim ve Gözlem Ormanı'dır. Yaralıgöz Eğitim ve Gözlem Ormanı, bitki coğrafyası açısından Avrupa-Sibirya ile İran-Turan floristik alanları arasındaki geçiş zonunda konumlanmaktadır.

Materyal ve Metod: Bu araştırma bir flora çalışması olup, materyalini 2011-2012 yılları arasında yapılan arazi çalışmaları sonucu toplanan damarlı bitkiler oluşturmaktadır.

Sonuçlar: Bu çalışma ile, 374 damarlı bitki tespit edilmiştir. Pteridophyta bölümü 6 takson, Pinidae alt-sınıfi 7 takson ve Magnoliidae alt-sınıfı ise 361 takson ile temsil edilmektedir. Çalışma alanında en geniş familya Asteraceae (45; \%12.1) olup bunu Lamiaceae (35; \%9.3), Rosaceae ve Fabaceae (27; \%7.2) familyaları takip etmektedir. Çalışma alanında tespit edilen bitki taksonlarından 190'ının fitocoğrafik bölgesi belirlenmiş ve bir bütün olarak değerlendirildiğinde Avrupa-Sibirya 144 (\%38.7), İran-Turan 24 (\%6.4) ve Akdeniz ise 22 (\%5.9) taksona sahiptir. Raunkier'in hayat formlarına göre Hemikriptofitler \%50.7, Fanerofitler \%15.7 ve Kriptofitler ise \%14.9 ile en sık görülen bitki formlarıdır.

Önemli vurgular: Çalışma sonucunda 23'ü endemik, 1'i nadir olmak üzere 24 tane tehlike altında olan bitki taksonu tespit edilmiştir. Bu endemik türlerden Acer hyrcanum subsp. keckianum ve Avrupa ladini taksonları için yeni yayılış alanları tespit edilmiştir. Birçok karakteristik bitki türünün varlığı nedeniyle, Yaralıgöz Eiğitim ve Gözlem Ormanı'nın önemli bir botanik turizm potansiyeline sahip olduğu belirlenmiştir.

Anahtar Kelimeler: Ekoturizm, flora, endemik, bitkisel biyoçeşitlilik, Türkiye, Yaralıgöz Dağı. 


\section{Introduction}

The concept of sustainability has emerged in order to sustain the existence of forest ecosystems within the balance of conservation-use (Kurtoğlu and Akbulut (2015). The concept of sustainability in forestry aims to provide various forest products and the social functions of the forest (Şen and Buğday, 2015). Developments in the world population and industry have led to an increase in demand for natural resource needs. This negative effect has reduced the productivity and continuity of many forest ecosystems (Özcan, 2016).

Tourism includes visits to different environments to meet the needs of people such as short-term travel, sight, recreation, and learning. Thirty-five years ago, a new concept emerged for human beings. This concept, called as nature tourism or ecotourism, has emerged based on the relationship between man and nature. Ecotourism is a kind of tourism that is sensitive to natural areas, protecting the environment and taking care of the welfare of the local people. Kurdoğlu (2002) describes ecotourism as the basis of the definition of International Union for Conservation of Nature (IUCN). He stated that environmentally sensitive tourism, by understanding nature and cultural resources, contribute to preserve the nature, has a low visitor impact and provides socio-economic benefits to local people.

Turkey has a magnificent botanic tourism potential assessed in terms of ecotourism (Anonymous, 2018). The flora (botanic) tourism, which constitutes a sub-section of ecotourism, is an alternative tourism branch, which has emerged in parallel with the increasing interest in biological diversity in recent years, and their protection and development (Irmak and Y1lmaz, 2011). Turkey, due to geographic location, topography, microclimate diversity, and the presence at the junction of three different floristic regions, is one of the most important centers in the world in terms of plant biodiversity by hosting many ecosystems (Terzioğlu, Bilgili \& Karaköse, 2009). As a result of this situation, of the 374000 (Christenhusz and Byng, 2016) plant species registered in the world, 12816 plant species are found within the borders of our country and the number of endemic plant taxa is known as 4040 (Güner, Aslan, Ekim, Vural \& Babaç, 2012; Özhatay, Kültür \& Gürdal, $2013 ; 2015 ; 2017)$. Botanic tourism isknown to provide contributions to regional economic development, providing four seasons of tourism, job opportunities (Ekim, 2002).

In Kastamonu province, one of the first places to come to mind when ecotourism tourism mentioned is Yaraligöz Mountain. The Regional Directorate of Forestry of Kastamonu (FRD) has planned a destination (Yaralıgöz Education and Observation Forest), for creating nature-based tourism in Yaralıgöz Mountain and its vicinity, in order to contribute to the tourism and the development of the province. Within in this destination, Kastamonu FRD has created different thematic areas such as biodiversity, ornithology, recreation areas, panoramic views, educational forest, and natural oldgrowth forests. In this context, the project was carried out to determine the plant biodiversity of Yaralıgöz EOF by a cooperation between Kastamonu FRD and Karadeniz Technical University Faculty of Forestry. This study constitutes only the flora level of the project.

\section{Material and Methods Study area}

This study covers part of the doctoral study of the author. Yaralıgöz EOF is located within the boundaries of Devrekani, Bozkurt and Çatalzeytin districts. Based on the planning unit, it remains within the four planning units (Devrekani, Şeyhşaban, Tezcan and Karacakaya) of the Kastamonu Forest Regional Directorate (Figure 1). 


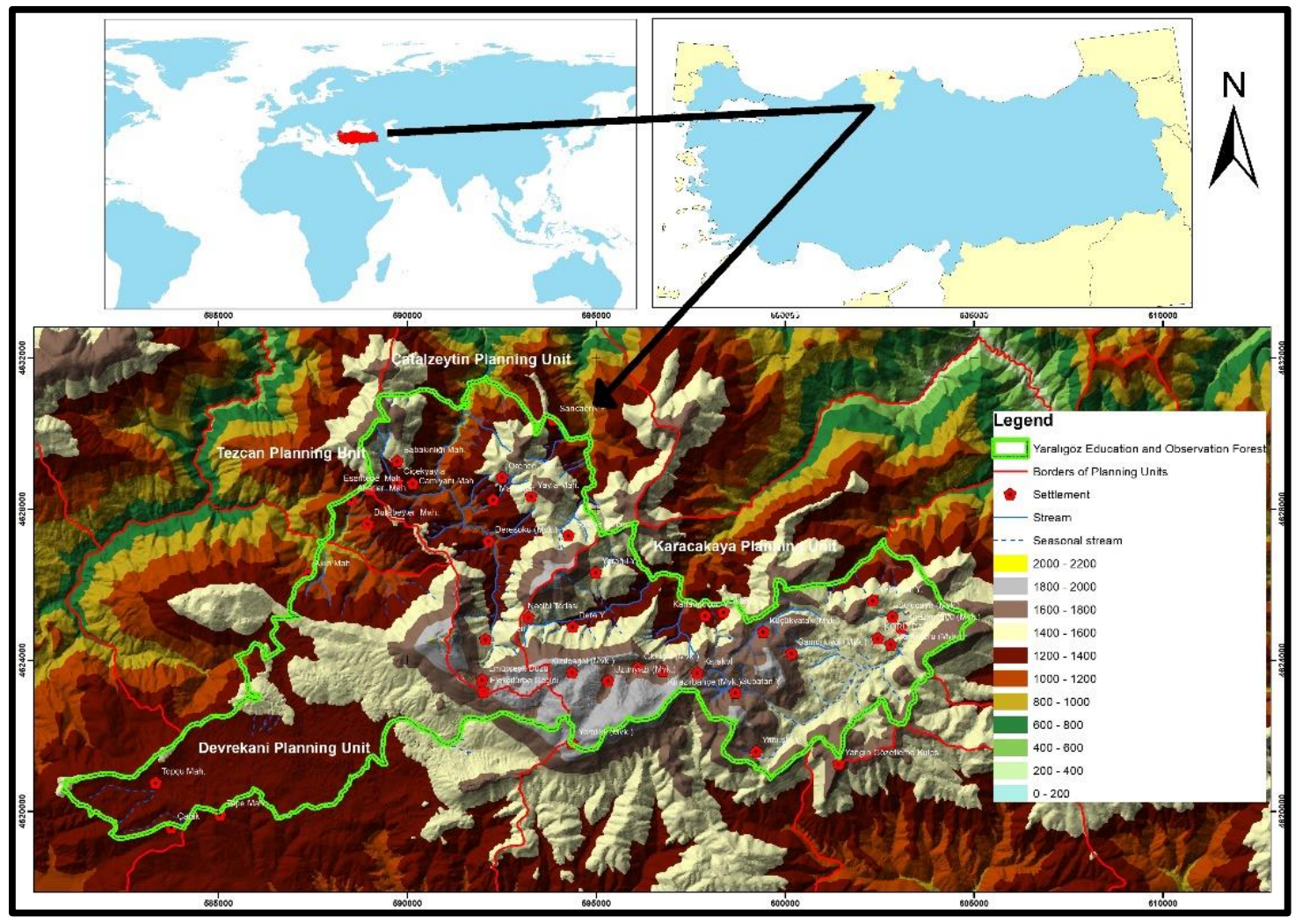

Figure 1. Location of the Yaralıgöz EOF

Yaralıgöz EOF is geomorphologically mountainous and steep land. As a geographical location, it is located at the foot of Yaralıgöz Mountain on the DevrekaniBozkurt road route in the northeast of Devrekani OPB and ends at Koru Mountain in the east. Yaraligöz EOF consists of a total area of $11.550,7$ ha, the altitude varies between $773 \mathrm{~m}$, and $2019 \mathrm{~m} .9800$ ha of this total area is composed of forest areas. In the study area, there are pure or mixed forests of Abies nordmanniana subsp. equi-trojani, Fagus orientalis, Pinus sylvestris, Pinus nigra, Carpinus betulus and Quercus petraea subsp. iberica species (Table 1). Common hornbeam dominated young forest is found southern part of the Yaraligöz Mountain at the Devrekani region. Sessile Oak forests are located in Devrekani and Şeyhşaban region, and Anatolian Black Pine forests are located in Devrekani and Tezcan region. Scots Pine forests spread on the eastern side of Yaralıgöz Mountain in the Karacakaya region with Juniperus. Oriental Beech and Kazdağ1 Fir forests have formed pure or mixed forests in the North-West-South direction of Yaralıgöz Mountain. The main streams in Yaralıgöz are Kabalaklı, Kayalıkoru, Üçgöller, Büyükçay, Görük, Süleyman Çayırı, Ark, Yanık Değirmen and Karasun. The main high points of the research area are; Türbekaya hill 2019 m, Dibekkorukayası $1860 \mathrm{~m}$, Büyükyazı hill $1832 \mathrm{~m}$, Koru mountain $1660 \mathrm{~m}$. The research area is located in the square A5 of Kastamonu province according to the system of Davis (1965-85). 
Table 1. Forest types in the Forest Planning Units

\begin{tabular}{lcccrr}
\hline Forests & Devrekani & Karacakaya & Şeyhşaban & Tezcan & Total (Ha) \\
\hline Beech & 25.7 & 2.7 & 344.3 & 28.6 & $401.3(5.1 \%)$ \\
\hline Beech-Fir & 291 & 244.8 & 599.9 & 79.1 & $1214.8(15.4 \%)$ \\
\hline Beech-Oak & & & 36.5 & & $36.5(0.5 \%)$ \\
\hline Oak & 16.9 & & 62.9 & 101.4 & $181.2(2.3 \%)$ \\
\hline Hornbeam dominated & 461 & 2.5 & 1.1 & & $464.6(5.9 \%)$ \\
\hline Fir & 1264.9 & 1961.6 & 905 & 34.5 & $4166.0(53 \%)$ \\
\hline Scots Pine & 127.2 & 832.8 & 202 & 44 & $1206.0(15.3 \%)$ \\
\hline Anatolian Black Pine & 114.5 & & & 19.6 & $134.1(1.7 \%)$ \\
\hline Scots Pine-Beech & & & 43.8 & 15.5 & $59.3(0.8 \%)$ \\
\hline Total (Ha) & 2301.2 & 3044.4 & 2195.5 & 322.7 & $7863.8(100 \%)$ \\
\hline
\end{tabular}

\section{Plant Materials and Identification}

The material of the study consists of plant samples obtained from field studies conducted between the years of 2011-2012. Plant samples were collected from the research area within the vegetation period in accordance with the plant collection rules. The location of the specimens collected during the field studies, the characteristics of the habitat, altitudes and collection dates were recorded. These plants were identified using the Flora of Turkey and the East Aegean Islands (Davis, 1965-85; Davis et al., 1988; Güner, Özhatay, Ekim \& Başer, 2000). In addition, various plant guides (Bonnier, 1912-1934; Phillips, 1994; Tekin, 2007a; 2007b) and plant samples of Karadeniz Technical University, faculty of forest herbarium (KATO) were used. Scientific and Turkish names of plant taxa were checked according to Turkish Plant List (Güner et al., 2012). Upper taxonomic units of plant taxa was prepared according to Christenhusz, Zhang, \& Schneider (2011a), for Pteridophyta; Christenhusz et al., (2011b) for Pinidae; and Angiosperm Phylogeny Group (APG) III (Stevens, 2001) for Magnoliidae.

\section{Result and Discussion}

After identification of plant specimens, 374 vascular plant taxa belonging to 71 families and 233 genera were found in Pteridophyta and Magnoliophyta (Pinidae and Magnoliidae) sections. Pteridophyta section was represented by $6(1.6 \%)$ taxa, Pinidae subclass by 7 (1.9\%), and Magnoliidae subclass by 361 (96.5\%) species and subspecies. Yaralıgöz EOF is located in the transitional zone between the Euxine province of Euro-Siberian and IranoTuranian floristic areas in terms of plant geography (Davis, Harper \& Hedge, 1971). The phytogeographical region of 190 $(50.57 \%)$ taxa in Yaralıgöz EOF was determined. The plant taxa classification in terms of phytogeographical regions was as follows: Euro-Siberian 105 (28.1\%), Euxine 29 (7.7\%), Euxine (mountain) 4 (1.1\%), Hyrcano-Euxine 6 (1.6\%), Irano-Turanian 24 (6.4\%), and Mediterranean $22(5.9 \%)$. The remaining 184 taxa $(49.2 \%)$ were cosmopolitan, multiregional or unknown regions (Table 2). Similar results were reported in previous studies in the Blacksea region (Eminağaoğlu and Anşin, 2004; Palabaş Uzun and Anşin, 2006; Uzun and Terzioğlu, 2008). In addition, the proportional distribution of determined taxa according to phytogeographic regions was compared with other studies close to the research area (Table 3). After the comparison, although the data obtained from the point of phytogeographical region are similar to some studies (Kılınç, 1985a; Kurt, 1992; Korkmaz and Engin, 2001; Özbek, 2004; Karaburç, 2006; Özen, Özbek \& Vural, 2013), it was found out that it is different from the existing other studies (Ketenoğlu and Güney, 1997; Kanoğlu, 2002; Baysal, 2008). The predominance of Euro-Siberian (consists of Euro-Siberian, Euxine, Euxine (mountain)) plant taxa is clearly prevailing in the research area. Additionally, Irano-Turanian (24 taxa-6.4\%) and Mediterranean (22 taxa-5.9\%) plants were observed.This situation arises from the 
climate characteristics and location of the study area. The northernmost part of the study area is limited with the Blacksea. According to Yurdakulol, Demirörs \& Yıldız, (2002) and Kanoğlu (2002), in the northern part of the area near the sea level (Abana-Çatalzeytin), there is maquis vegetation, which is one of the characteristic vegetation types of the Mediterranean floristic region. This influenced the spread of Mediterranean plants into Yaralıgöz EOF. Another factor is the characteristic climate of the area, which is in transition to the continental climate type in Devrekani, which is part to the south of the area. This situation has allowed the coexistence of Pinus nigra and Irano-Turanian elements.

Table 2. Numerical and proportional distributions of taxa according to phytogeographical regions

\begin{tabular}{lcc}
\hline $\begin{array}{c}\text { Phytogeographical } \\
\text { region }\end{array}$ & $\begin{array}{c}\text { Number } \\
\text { of taxa }\end{array}$ & Rates (\%) \\
\hline Euro-Siberian & 105 & 28.1 \\
\hline Euxine & 29 & 7.7 \\
\hline Euxine (mt) & 4 & 1.1 \\
\hline Hyrcano-Euxine & 6 & 1.6 \\
\hline Irano-Turanian & 24 & 6.4 \\
\hline Mediterranean & 15 & 4 \\
\hline East Mediterranean & 6 & 1.6 \\
\hline East Mediterranean $(\mathrm{mt})$ & 1 & 0.3 \\
\hline Others & 184 & 49.2 \\
\hline Total & 374 & 100 \\
\hline
\end{tabular}

Table 4 shows the numerical and proportional distributions of the prominent taxa in terms of taxon richness from the 71 families determined in the research area. These families were compared with the studies conducted in the adjacent areas. According to the number of taxa from the families in the study area, Asteraceae family stands out. This situation can be explained by the fact that the Asteraceae is the richest family in terms of the number of taxa on earth, and the taxa have a wide ecological amplitude and long-distance distribution of their diaspores. 
Table 3. Comparison of plant taxa in terms of phytogeographic region and endemism

\begin{tabular}{|c|c|c|c|c|c|c|c|c|c|c|c|c|c|c|c|c|c|c|c|c|}
\hline \multirow[t]{2}{*}{$\begin{array}{l}\text { Phytogeographic } \\
\text { region and } \\
\text { endemism }\end{array}$} & \multicolumn{2}{|c|}{$\begin{array}{l}\text { Present } \\
\text { Study }\end{array}$} & \multicolumn{2}{|c|}{$\begin{array}{l}\text { Kanoğlu } \\
2002 \\
\text { (Abana) }\end{array}$} & \multicolumn{2}{|c|}{$\begin{array}{c}\text { Özen et al. } \\
2013 \\
\text { (Armutluçayır) }\end{array}$} & \multicolumn{2}{|c|}{$\begin{array}{c}\text { Özbek } \\
2004 \\
\text { (Kurtgirmez) }\end{array}$} & \multicolumn{2}{|c|}{$\begin{array}{c}\text { Ketenoğlu } \\
\text { and Güney } \\
1997 \text { (Bat1 } \\
\text { Küre) }\end{array}$} & \multicolumn{2}{|c|}{$\begin{array}{l}\text { Karaburç } \\
2006 \\
\text { (Oyrak) }\end{array}$} & \multicolumn{2}{|c|}{$\begin{array}{c}\text { Kurt } \\
1992 \\
\text { (Köklüce) }\end{array}$} & \multicolumn{2}{|c|}{$\begin{array}{c}\text { Baysal } \\
2008 \\
\text { (Çangal) }\end{array}$} & \multicolumn{2}{|c|}{$\begin{array}{c}\text { Korkmaz } \\
\text { and Engin } \\
2001 \\
\text { (Boyabat) }\end{array}$} & \multicolumn{2}{|c|}{$\begin{array}{c}\text { Kilınç } \\
\text { 1985a } \\
\text { (Devrez) }\end{array}$} \\
\hline & No. & $(\%)$ & No. & $(\%)$ & No. & $(\%)$ & No. & $(\%)$ & No. & $(\%)$ & No. & $(\%)$ & No. & $(\%)$ & No. & $(\%)$ & No. & $(\%)$ & No. & $(\%)$ \\
\hline Euro-Siberian & 144 & 38,7 & 118 & 32,1 & 103 & 37,2 & 136 & 31,6 & 259 & 41,8 & 38 & 14,6 & 82 & 33,3 & 230 & 30 & 172 & 16,9 & 202 & 23,8 \\
\hline Irano-Turanian & 24 & 6,4 & 2 & 0,5 & 6 & 2,2 & 23 & 5,3 & 50 & 8 & 33 & 13 & 26 & 10,6 & 46 & 6 & 115 & 11,3 & 140 & 16,5 \\
\hline Mediterranean & 22 & 5,9 & 34 & 9,2 & 3 & 1,1 & 16 & 3,7 & 88 & 14,4 & 25 & 9,6 & 16 & 6,5 & 48 & 6,2 & 99 & 9,7 & 80 & 9,5 \\
\hline Endemic & 23 & 6,4 & 5 & 1,4 & 9 & 3,2 & 34 & 7,9 & 44 & 7,2 & 23 & 8,8 & 35 & 14,2 & 56 & 7,3 & 106 & 10,4 & 16 & 2 \\
\hline Total & \multicolumn{2}{|c|}{374} & \multicolumn{2}{|c|}{371} & \multicolumn{2}{|c|}{277} & \multicolumn{2}{|c|}{431} & \multicolumn{2}{|c|}{613} & \multicolumn{2}{|c|}{260} & \multicolumn{2}{|c|}{246} & \multicolumn{2}{|c|}{769} & \multicolumn{2}{|c|}{1016} & \multicolumn{2}{|c|}{850} \\
\hline
\end{tabular}

Table 4. Comparison of the families containing the most taxa in previous studies conducted in nearby areas.

\begin{tabular}{|c|c|c|c|c|c|c|c|c|c|c|c|c|c|c|c|c|c|c|}
\hline \multirow[t]{2}{*}{ Families } & \multicolumn{2}{|c|}{$\begin{array}{c}\text { Present } \\
\text { Study }\end{array}$} & \multicolumn{2}{|c|}{$\begin{array}{l}\text { Kanoğlu } \\
2002 \\
\text { (Abana) }\end{array}$} & \multicolumn{2}{|c|}{$\begin{array}{c}\text { Özen et al. } \\
2013 \\
\text { (Armutluçayır) }\end{array}$} & \multicolumn{2}{|c|}{$\begin{array}{c}\text { Özbek } \\
2004 \\
\text { (Kurtgirmez) }\end{array}$} & \multicolumn{2}{|c|}{$\begin{array}{c}\text { Ketenoğlu } \\
\text { and Güney } \\
1997 \text { (Bat1 } \\
\text { Küre) }\end{array}$} & \multicolumn{2}{|c|}{$\begin{array}{c}\text { Özen and } \\
\text { Kılınç } 1995 \\
\text { (Alaçam- } \\
\text { Gerze) }\end{array}$} & \multicolumn{2}{|c|}{$\begin{array}{c}\text { Baysal } \\
2008 \\
\text { (Çangal) }\end{array}$} & \multicolumn{2}{|c|}{$\begin{array}{c}\text { Karaburç } \\
2006 \\
\text { (Oyrak) }\end{array}$} & \multicolumn{2}{|c|}{$\begin{array}{c}\text { Kurt } \\
1992 \\
\text { (Köklüce) }\end{array}$} \\
\hline & No. & $(\%)$ & No. & $(\%)$ & No. & $(\%)$ & No. & $(\%)$ & No. & $(\%)$ & No. & $(\%)$ & No. & $(\%)$ & No. & $(\%)$ & No. & $(\%)$ \\
\hline Asteraceae & 45 & 12.1 & 40 & 10.8 & 35 & 12.6 & 28 & 9.8 & 60 & 9.7 & 72 & 10.4 & 92 & 11.9 & 31 & 12 & 26 & 10.7 \\
\hline Lamiaceae & 35 & 9.3 & 26 & 7 & 20 & 7.2 & 18 & 6.3 & 38 & 6.1 & 40 & 5.8 & 57 & 7.4 & 25 & 10 & 23 & 9.5 \\
\hline Rosaceae & 27 & 7.2 & 18 & 4.9 & 20 & 7.2 & 17 & 5.9 & 27 & 4.3 & 24 & 4.5 & 34 & 4.4 & 13 & 5 & 17 & 7 \\
\hline Fabaceae & 27 & 7.2 & 27 & 7.3 & 17 & 6.1 & 19 & 6.6 & 63 & 10.2 & 68 & 9.9 & 59 & 7.7 & 23 & 9 & 28 & 11.4 \\
\hline Poaceae & 13 & 3.5 & 11 & 3 & 20 & 7.2 & 15 & 5.2 & 31 & 5 & 49 & 7.1 & 28 & 3.6 & 10 & 4 & 16 & 6.6 \\
\hline Orchidaceae & 12 & 3.2 & 10 & 2.7 & 2 & 0.5 & 6 & 1.4 & 14 & 2.3 & 17 & 2.5 & 17 & 2.2 & 3 & 1.2 & 8 & 3.3 \\
\hline Caryophyllaceae & 11 & 2.9 & 7 & 1.9 & 5 & 1.3 & 12 & 2.9 & 24 & 3.8 & 23 & 3.3 & 26 & 3.4 & 9 & 3.5 & 9 & 3.7 \\
\hline Plantaginaceae & 11 & 2.9 & 2 & 0.5 & 1 & 0.3 & 1 & 0.2 & 2 & 0.3 & 4 & 0.6 & 2 & 0.3 & 1 & 0.4 & 1 & 0.4 \\
\hline Boraginaceae & 10 & 2.7 & 7 & 1.9 & 9 & 2.4 & 11 & 3.8 & 20 & 3.3 & 19 & 2.7 & 24 & 3.1 & 10 & 4 & 5 & 2.1 \\
\hline Brassicaceae & 8 & 2.1 & 10 & 2.7 & 15 & 4 & 19 & 6.6 & 28 & 4.6 & 22 & 3.9 & 41 & 5.3 & 15 & 6 & 5 & 2.1 \\
\hline Total & \multicolumn{2}{|c|}{374} & \multicolumn{2}{|c|}{371} & \multicolumn{2}{|c|}{277} & \multicolumn{2}{|c|}{431} & \multicolumn{2}{|c|}{613} & \multicolumn{2}{|c|}{696} & \multicolumn{2}{|c|}{76} & \multicolumn{2}{|c|}{260} & \multicolumn{2}{|c|}{246} \\
\hline
\end{tabular}


Of the plants identified in the Yaralıgöz area, 23 were endemic (14\%) and the endemism rate was $6.4 \%$ (Table 5). This rate is considerably lower than the national average. This low endemism ratio is parallel with the fact that the study area is located in the European-Siberian floristic region (Avc1, 2005). With this study, new distribution areas were determined for two plant taxa. First takson is Acer hyrcanum subsp. keckianum, which is endemic to Turkey. According to flora of Turkey, the distribution areas of this woody species were B1 Balıkesir, B2 Kütahya and B3 Afyon regions (Yaltırı, 1967). With this study, the first record of this woody species was made for the A5 Kastamonu province. The other plant species is Picea abies, which generally used as ornamental tree in our country. This tree species has found in a forest standbecause of accidental use of individuals belonging to Picea abies in a Pinus sylvestris afforestation study carried out around Koru Mountain about 30 years ago.

Table 5. Endemic and rare plants of the Yaralıgöz EOF

\begin{tabular}{|c|c|c|c|}
\hline Number & Taxon & Endemic/Rare & IUCN Category \\
\hline 1 & Erodium birandianum & Endemic & EN \\
\hline 2 & Lilium martagon & Rare & VU \\
\hline 3 & Tragopogon dshimilensis & Endemic & VU \\
\hline 4 & Crocus speciosus subsp. ilgazensis & Endemic & NT \\
\hline 5 & Sempervivum gilliani & Endemic & NT \\
\hline 6 & Abies nordmanniana subsp. equi-trojani & Endemic & $\mathrm{LC}$ \\
\hline 7 & Acer hyrcanum subsp. keckianum & Endemic & $\mathrm{LC}$ \\
\hline 8 & Astragalus densifolius subsp. amasiensis & Endemic & $\mathrm{LC}$ \\
\hline 9 & Asyneuma limonifolium subsp. pestalozzae & Endemic & $\mathrm{LC}$ \\
\hline 10 & Centaurea urvillei subsp. stepposa & Endemic & $\mathrm{LC}$ \\
\hline 11 & Cyanus reuterianus var. phrygia & Endemic & $\mathrm{LC}$ \\
\hline 12 & Dianthus leucophaeus & Endemic & $\mathrm{LC}$ \\
\hline 13 & Euonymus latifolius subsp. cauconis & Endemic & $\mathrm{LC}$ \\
\hline 14 & Helichrysum arenarium subsp. aucheri & Endemic & $\mathrm{LC}$ \\
\hline 15 & Linum olympicum & Endemic & $\mathrm{LC}$ \\
\hline 16 & Lonicera orientalis & Endemic & $\mathrm{LC}$ \\
\hline 17 & Onosma bornmuelleri & Endemic & $\mathrm{LC}$ \\
\hline 18 & Onosma isauricum & Endemic & $\mathrm{LC}$ \\
\hline 19 & Salvia cyanescens & Endemic & $\mathrm{LC}$ \\
\hline 20 & Sideritis germanicopolitana subsp. germanicopolitana & Endemic & $\mathrm{LC}$ \\
\hline 21 & Stachys setifera subsp. lycia & Endemic & $\mathrm{LC}$ \\
\hline 22 & Trifolium elongatum & Endemic & $\mathrm{LC}$ \\
\hline 23 & Veronica multifida & Endemic & $\mathrm{LC}$ \\
\hline 24 & Vicia freyniana & Endemic & $\mathrm{LC}$ \\
\hline
\end{tabular}

The life forms of plant taxa were determined according to Raunkiaer (1934). The distribution of plant species respect to life forms is as follows; Hemicryptophytes 190 (50.7\%), Phanerophytes 59 (15.7\%), Cryptophytes 56 (14.9\%), Therophytes 43
(11.7\%), Chamaephytes $24 \quad(6.4 \%)$ and Vascular Parasite $2(0.5 \%)$. The dominance of Hemicryptophytes indicates a cold, humid and semi-humid climate in the study area (Raunkiaer, 1934). A similar pattern was found in the Tosya and Devrez regions 
(Kilıç, 1985b). There is an increase in the number of phanerophytes and cryptophytes with the effect of the phytogeographic region and altitude in the study area.(Naqinezhad, Zare-Maivan \& Gholizadeh, 2015; Cain, 1950). The fact that the Phanerophyte plant taxa in the study area is $15.7 \%$ indicates that Yaralıgöz region is very rich in woody vegetation. This result corresponds to the woody species richness criterion, which is one of the reasons, for the creation of the Yaralıgöz EOF.. Eminağaoğlu and Akyıldırım (2015), in their study on plant richness of Artvin province, have mentioned the importance of woody species in terms of botanic tourism. It is possible to see very different forest ecosystems formed by woody species. While most of these forest ecosystems are formed by Abies nordmanniana subsp. equi-trojani, Fagus orientalis and Pinus sylvestris, there are forest communities formed by Pinus nigra, Quercus petraea subsp. iberica and Carpinus betulus. Many woody species, which are of great importance in terms of both wildlife and ecosystem balance, are able to regenerate naturally. Corylus colurna, Taxus baccata, Rhododendron luteum, Laurocerasus officinalis, Rosa canina, Sorbus aucuparia, Berberis vulgaris, Mespilus germanica, Rubus idaeus, Salix caprea, Populus tremula, Juniperus communis, Juniperus oxycedrus are some of these species. In addition, this situation provides different phenotypic aspect during different seasons and attracts the interest of nature lovers. Botanic tourism is considered to serve the special biological interests of individual tourists and groups (Trauer, 2006). Irmak and Yilmaz (2011) identified the priorities for the botanic tourism. Finally, special interest groups were categorized according to the participants' answers. Aromatic plants are the first preference in this order, followed by geophytes, endemic, woody and medicinal plants. There is special demand from these special interest groups, which have established various associations focusing on Cyclamens, orchids, medicinal and aromatic plants, dendrology, succulents(Eminağaoğlu and Akyıldırım, 2015). Yaralıgöz EOF has a significant potential harbouring 87 medicinal and aromatic plants (Karaköse, 2015) including Alchemilla mollis, Hedera helix, Hypericum perforatum, Laurocerasus officinalis, Rubus hirtus, Rubus ideaus, Rosa canina, Thymus sipyleus, Urtica dioica and Trachystemon orientalis. Kastamonu and the surrounding areas are important sahlep-tuber production areas (Yaman and Akyıldız, 2008). As mentioned in the life forms section of plant species, the number of geophytes is high. Plant species belonging to Dactylorhiza, Orchid, Anacamptis, Colchicum, Fritillaria, Lilium, Crocus, Allium, Sedum, Sempervivium, which are distributed naturally in Yaralıgöz EOF, meet the demands of these interest groups. Another important issue in botanic tourism is the appropriate excursion time and route for interest groups (Aklıbaşında, Bulut \& Külekçi, 2012). For Yaralıgöz region, it was found out that the optimum excursion time was between May and July. During this period, it is possible to see most plant species except some special plants (flowering plants at spring and autumn). The Kastamonu FRD installed a suitable the excursion route. It starts from Emürseyit, which is close to the summit of Yaralıgöz Mountain, and reaches Koru Mountain in the east. Along the walking route, it is possible to see pure or mixed forests formed by Pinus sylvestris, Fagus orientalis and Abies nordmanniana species. Additionally, there are many medical and aromatic, endemic, geophyte and woody plants to be seen. This excursion route also includes a locality for the Erodium birandianum, which is endemic to Turkey. 


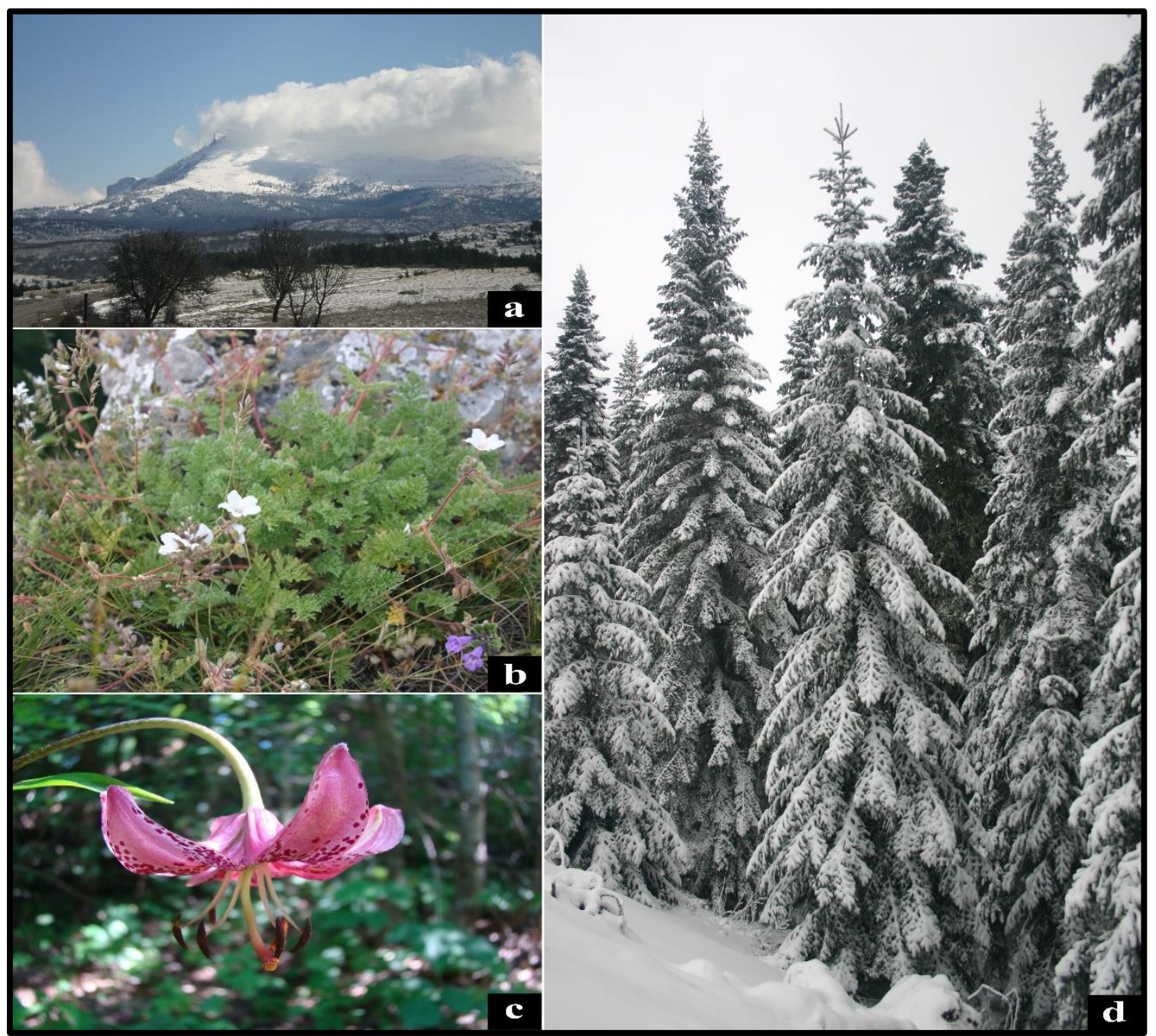

Figure 2. Yaralıgöz Mountain (a) and three remarkable plant taxa; b) Erodium birandianum, c) Lilium martagon, d) Abies nordmanniana subsp. equi-trojani, ; 


\section{Conclusions}

The Western Black Sea Region has a rich woody flora due to its various vegetation types (forest, riparian, wetland, alpinesubalpin, pseudo maquis, maquis and sand dunes). This is reflected in the rich flora of Yaralıgöz Education and Observation Forest. This richness should be included in planning (ecotourism, forestry and wildlife). It is necessary to prioritize natural species in afforestation works and recognize their multifunctional ecosystem services.

Botanic tourism has the potential to contribute to the economic development of our country, regions and provinces. Without proper management, botanic tourism may have negative impacts such as careless behaviors, damages to the target plant species, habitat destruction, as well as biosmuggling cases, whose awareness has increased in recent years. It is necessary to know the botanic and flora tourism preferences have both the economic contributions and the some negative impacts. For that reason, some preventive measures need to be taken by Kastamonu FRD for sustainable management in Yaralıgöz EOF.

Vascular Plants to be seen in terms of botanic tourism in the Yaralıgöz EOF.;

Pteridophyta Division

Polypodiidae (Fern Subclass)

1. Dennstaedtiaceae

1. Pteridium aquilinum (L.) Kuhn. -(Kartal Eğreltisi), A5, KATO: 19691; C.

2. Cystopteridaceae

2. Cystopteris fragilis (L.) Bernh. -(Gevrek Eğrelti), A5, KATO: 19692; C.

3. Aspleniaceae

3. Asplenium trichomanes L. -(Saçakotu), A5, KATO: 19693; C.

4. Dryopteridaceae

4. Dryopteris affinis (Lowe) Fraser-Jenkins subsp. borreri (Newm.) Fraser-Jenkins (Geyik Piluncu), A5, KATO: 19694; C.

5. D. filix-mas (L.) Schott. -(Erkek Eğrelti), A5, KATO: 19695; C.

6. Polystichum lonchitis (L.) Roth -(Uzun Pilunç), A5, KATO: 19696; C.

\section{Magnoliophyta Division \\ Pinidae (Pine subclass)}

5. Pinaceae

7. Abies nordmanniana (Stev.) Spach subsp. equi-trojani (Asc. \& Sint. ex Boiss.) Coode \& Cullen -(Kazdağı Göknarı), A5: KATO: 19697; Eux., End., Ph.

8. Picea abies (L.) Karst. -(Avrupa Ladini), A5, KATO: 19698; Ph.

9. Pinus nigra J. F. Arnold subsp. pallasiana (Lamb.) Holmboe var. pallasiana (Karaçam), A5, KATO: 19699; Ph.

10. P. sylvestris L. var. hamata Steven (Sarıçam), A5, KATO: 19700; Euro-Sib., Ph.

6. Cupressaceae

11. Juniperus communis L. var. saxatilis Pall. -(Adi Ardıç), A5, KATO: 19701; Ph.

12. J. oxycedrus L. subsp. oxycedrus (Katran Ardic1), A5, KATO: 19702; Ph.

7. Taxaceae

13. Taxus baccata L. -(Adi Porsuk), A5, KATO: 19703; Ph.

\section{Magnoliidae (Magnolia subclass)}

8. Araceae Juss.

14. Arum elongatum Steven -(Y1lancücüğü), A5, KATO: 19704; C.

9. Colchicaceae DC.

15. Colchicum speciosum Steven -(Şepart), A5, KATO: 19705; Eux., C.

16. C. umbrosum Steven -(Şaşortkovan), A5, KATO: 19706; Eux., C.

10. Liliaceae Juss.

17. Fritillaria pinardii Boiss. -(Mahçup Lale), A5, KATO: 19707; Ir.-Tur., C.

18. Gagea villosa (Bieb.) Duby var. villosa(Tüylü Yıldız), A5, KATO: 19708; Medit., C.

19. Lilium martagon L. -(Sultan Zambağ 1$)$, A5, KATO: 19709; Euro.-Sib., Rare, C.

12. Orchidaceae Juss.

20. Anacamptis pyramidalis (L.) Rich. (Sivri Salep), A5, KATO: 19710; C.

21. Cephalanthera damasonium (Mill.) Druce -(Orman Kuşcuğu), A5, KATO: 19711; Euro.-Sib., C.

22. Dactylorhiza romana (Seb.) Soò subsp. romana-(Elçik), A5, KATO: 19712; Medit., C.

23. D. urvilleana (Steudel) Baumann \& Künkele subsp. urvilleana-(Balkaymak), A5, KATO: 19713; Eux., C. 
24. Epipactis helleborine (L.) Crantz subsp. helleborine -(Bindallıçiçeği), A5, KATO: 19714; C.

25. Limodorum abortivum (L.) Sw. var. abortivum -(Saçuzatan), A5, KATO: 19715; C.

26. Orchis anatolica Boiss. -(Anadolu Orkidesi), A5, KATO: 19716; Medit., C.

27. O. morio L. subsp. morio -(Gelincik Salebi), A5, KATO: 19717; Medit., C.

28. O. pallens L. -(Solgun Salep), A5, KATO: 19718; Euro.-Sib., C.

29. O. purpurea Hudson subsp. purpurea(Hasanc1k), A5, KATO: 19719; Euro.-Sib., C.

30. O. simia Lam. -(Salep Püskülü), A5, KATO: 19720; Medit., C.

31. Serapias orientalis (Greuter) H.Baumann \& Künkele subsp. orientalis-(Dillikulak), A5, KATO: 19721; E. Medit., C.

13. Iridaceae Juss.

32. Crocus speciosus Bieb. subsp. ilgazensis Mathew -(Ilgaz Çiğdemi), A5, KATO: 19722; Euro.-Sib., End., C.

33. Allium carinatum L. subsp. carinatum (Sirtlı Körmen), A5, KATO: 19723; C.

34. A. scorodoprasum L. subsp. jajlae (Vved.) Stearn -(Deli Prrasa), A5, KATO: 19724; Eux., C.

35. A. scorodoprasum L. subsp. rotundum (L.) Stearn -(Deli P1rasa), A5, KATO: 19725; Eux, C.

36. A. pseudoflavum Steven -(Küllü Soğan), A5, KATO: 19726; Ir.-Tur., C.

37. A. rupestre Steven -(Taş Körmeni), A5, KATO: 19727; Eux., C.

14. Asparagaceae Juss.

38. Muscari armeniacum Leichtlin ex Baker -(Gavurbaşı), A5, KATO: 19728; C.

39. M. comosum (L.) Mill. -(Morbaş), A5, KATO: 19729; Medit., C.

40. Ornithogalum oligophyllum E. D. Clarke -(Kurt Soğanı), A5, KATO: 19730; C.

41. Polygonatum multiflorum Desf. (Boğumluca), A5, KATO: 19731; Eux., C.

42. Scilla bifolia L. -(Orman Sümbülü), A5, KATO: 19732; Medit., C.

15. Juncaceae Juss.

43. Juncus articulatus L. subsp. articulatus (Camışotu), A5, KATO: 19733; C.

44. J. inflexus L. subsp. inflexus -(Sazak), A5, KATO: 19734; C.

16. Cyperaceae Juss.
45. Blysmus compressus (L.) Panz. ex Link subsp. compressus -(Yass1 Hasirotu), A5, KATO: 19735; C.

46. Carex flacca Schreb subsp. erythrostachys (Hoppe) Holub -(Yanık Çayırsazı), A5, KATO: 19736; Medit., H.

47. C. sylvatica Huds. subsp. sylvatica (Mera Sazı), A5, KATO: 19737; Euro.-Sib., $\mathrm{H}$.

48. Eleocharis quinqueflora (Hartmann) O. Schwarz -(Seyreksaz), A5, KATO: 19738; C.

17. Poaceae Barnhart

49. Agrostis capillaris L. var. capillaris (Karahasanotu), A5, KATO: 19739; Euro.Sib., H.

50. Avena fatua L. var. fatua -(Deli Yulaf), A5, KATO: 19740; Th.

51. Briza media L. -(Zembilotu), A5, KATO: 19741; Th.

52. Bromus tomentellus Boiss. -(Bozkır Bromu), A5, KATO: 19742; Ir.-Tur., Th.

53. Dactylis glomerata L. subsp. glomerata(Domuz Ayrığı), A5, KATO: 19743; Euro.Sib., $\mathrm{H}$.

54. Festuca drymeja Mertens \& Koch -(Çalı Yumağı), A5, KATO: 19744; Euro.-Sib., H. 55. Hordeum bulbosum L. -(Boncuk Arpa), A5, KATO: 19745; $\mathrm{H}$.

56. Lolium perenne L. -(Çim), A5, KATO: 19746; Euro.-Sib., H.

57. Milium vernale $\mathrm{M}$. Bieb. subsp. vernale (Narin Darı), A5, KATO: 19747; Medit., Th. 58. Phleum exaratum Hochst. ex Griseb. (Meşe İtkuyruğu), A5, KATO: 19748; Th.

59. Poa pratensis L. -(Çayır Salkımotu), A5, KATO: 19749; $\mathrm{H}$.

60. P. trivialis L. -(Kaba Salkımotu), A5, KATO: $19750 ; \mathrm{H}$.

61. Stipa lessingiana Trin. \& Rupr. -(Gevşek Sorguçotu), A5, KATO: 19751; H.

18. Papaveraceae Juss.

62. Corydalis cava (L.) Schw. \& Körte subsp. cava -(Çayır kazgagası), A5, KATO: 19752; C.

63. Papaver dubium L. subsp. dubium (Köpekyağı), A5, KATO: 19753; Th.

64. P. rhoeas L. -(Gelincik), A5, KATO: 19754; Th.

19. Berberidaceae Juss.

65. Berberis crataegina DC. -(Karamuk), A5, KATO: 19755; Ir.-Tur., Ph.

20. Ranunculaceae Juss. 
66. Clematis vitalba L. -(Ak asma), A5, KATO: 19756; Ch.

67. Helleborus orientalis Lam. -(Noel gülü), A5, KATO: 19757; Eux., H.

68. Ranunculus brutius Ten. -(Buladan otu), A5, KATO: 19758; Euro.-Sib., C.

69. R. illyricus L. subsp. illyricus -(Gümüş düğünçiçeği), A5, KATO: 19759; C.

70. $R$. repens L. -(Tiktakdana), A5, KATO: 19760; $\mathrm{H}$.

21. Paeoniaceae Raf.

71. Paeonia peregrina Mill. -(Bocur), A5, KATO: $19761 ; \mathrm{H}$.

22. Crassulaceae J. St.-Hil.

72. Phedimus stoloniferus (S.G.Gmel.) Hart -(Pisikulağı), A5, KATO: 19762; Hyr.-Eux., Ch.

73. Sedum acre L. subsp. acre -(Ac1 Damkoruğu), A5, KATO: 19763; Ch.

74. S. pallidum Bieb. -(Koyunörmece), A5, KATO: 19764; Ch.

75. Sempervivum gillianiae Muirhead (Arzuotu), A5, KATO: 19765; Eux. (mt), End., Ch.

23. Saxifragaceae Juss.

76. Saxifraga cymbalaria L. -(Sar1 Taşkıran), A5, KATO: 19766; Th.

77. S. rotundifolia L. subsp. rotundifolia (Benli Taşkıran), A5, KATO: 19767; Euro.Sib., H.

24. Santalaceae R. Br.

78. Thesium arvense Horv. -(Tez Güvelek), A5, KATO: 19768; Euro.-Sib., H.

79. Viscum album L. subsp. album (Ökseotu), A5, KATO: 19769; VP.

25. Polygonaceae Juss.

80. Polygonum lapathifolium L. -(Tirşon), A5, KATO: 19770; Th.

81. Rumex obtusifolius L. subsp. subalpinus (Schur) Celak -(Kökükızıl), A5, KATO: 19771; H.

82. R. tuberosus L. subsp. horizontalis (Koch.) Rech. -(Kömeturşusu), A5, KATO: 19772; C.

26. Caryophyllaceae Juss.

83. Cerastium chlorifolium Fisch. \& C.A.Mey. -(Parlak Boynuzotu), A5, KATO: 19773; Th.

84. Dianthus calocephalus Boiss. -(Güzel Karanfil), A5, KATO: 19774; Ch.

85. D. leucophaeus Sm. -(Hoş Karanfil), A5, KATO: 19775; End., Ch.
86. Minuartia juniperina (L.) Marie \& Petitm. -(Hanım Şiltesi), A5, KATO: 19776; Ch.

87. Moenchia mantica (L.) Bartl. -(Dördüz otu), A5, KATO: 19777; Th.

88. Silene compacta Fisch. ex Hornem. (Kanlıbasira Otu), A5, KATO: 19778; H.

89. S. dichotoma subsp. racemosa (Otth) Graebn. \& P.Graebn. -(Salkım Nak11), A5, KATO: 19779; H.

90. S. italica (L.) Pers. subsp. italica (Yuğuş Yüreği), A5, KATO: 19780; Medit., $\mathrm{H}$.

91. S. latifolia Poir. subsp. eriocalycinae (Boiss.) Greuter \& Burdet -(Gicime), A5, KATO: $19781 ; \mathrm{H}$.

92. S. vulgaris (Moench.) Garcke var. vulgaris -(Ecibücü), A5, KATO: 19782; H.

93. Stelleria media (L.) Vill. -(Kuşotu), A5, KATO: 19783; Th.

27. Celastraceae R. Br.

94. Euonymus latifolius (L.) Mill. subsp. cauconis Coode \& Cullen -(İşyanotu), A5, KATO: 19784; Euro.-Sib., End., Ph.

95. E. latifolius (L.) Mill. subsp. latifolius (İğağac1), A5, KATO: 19785; Euro.-Sib., Ph.

28. Oxalidaceae R. Br.

96. Oxalis acetosella L. -(Ekşiyonca), A5, KATO: 19786; H.

29. Euphorbiaceae Juss.

97. Euphorbia amygdaloides L. var. amygdaloides -(Zerana), A5, KATO: 19787; Euro.-Sib., H.

98. E. macroclada Boiss. -(Neblul), A5, KATO: 19788; Ir.-Tur., H.

99. E. seguieriana Necker subsp. seguieriana -(Tasmaotu), A5, KATO: 19789; Ch.

100. E. stricta L. -(Kat1 Sütleğen), A5, KATO: 19790; Euro.-Sib., Th.

101. Mercurialis perennis L. -(Köpek Marulu), A5, KATO: 19791; Euro.-Sib., H.

30. Hypericaceae Juss.

102. Hypericum bithynicum Boiss. -(Uludağ Koyunkıran1), A5, KATO: 19792; Eux., H. 103. H. montbretii Spach -(Çay Kantaronu), A5, KATO: 19793; $\mathrm{H}$.

104. H. orientale L. -(Sandık Çiçeği), A5, KATO: 19794; H.

105. $H$. perforatum $\mathrm{L}$. subsp. veronense (Schrank) H. Linb. -(Kantaron), A5, KATO: 19795; H.

31. Linaceae DC. ex Perleb 
106. Linum austriacum L. subsp. glaucescens (B) Davis -(Puslu Zeyrek), A5, KATO: 19796; H.

107. L. olympicum Boiss. -(Uludağ Keteni), A5, KATO: 19797; End., H.

108. L. usitatissimum L. -(Keten), A5, KATO: 19798; Th.

32. Salicaceae Mirb.

109. Salix caprea L. -(Keçi Söğüdü), A5, KATO: 19799; Euro.-Sib., Ph.

110. S. cinerea L. var. cinerea -(Boz Söğüt), A5, KATO: 19800; Euro.-Sib., Ph.

111. S. elaeagnos Scop. -(İğde Söğüdü), A5, KATO: 19801; Euro.-Sib., Ph.

112. Populus tremula L. -(Titrek Kavak), A5, KATO: 19802; Euro.-Sib., Ph.

33. Violaceae Batsch

113. Viola kitaibeliana Roem. \& Schult. (Yabani Menekşe), A5, KATO: 19803; Th.

114. V. sieheana Becker -(Çayır Menekşesi), A5, KATO: 19804; Th.

34. Fabaceae Lindl.

115. Anthyllis vulneraria L. subsp. boissieri (Sag.) Bornm. -(Çobangülü), A5, KATO: 19805; H.

116. Argyrolobium biebersteinii Ball -(Ac1 Collik), A5, KATO: 19806; $\mathrm{H}$.

117. Astragalus angustifolius Lam. subsp. pungens (Willd.) Hayek -(Kör Geven), A5, KATO: 19807; Ch.

118. A. densifolius Lam. subsp. amasiensis (Freyn) Aytaç \& Ekim -(Amasya Gümüşü), A5, KATO: 19808; End., Ch.

119. A. glycyphylloides DC. -(Tatl1 Geven) A5, KATO: 19809; Euro.-Sib., H.

120. A. strigillosus Bunge -(Sert Geven), A5, KATO: 19810; Ir.-Tur., H.

121. Cytisus pygmaeus Willd. -(Cüce Keçitırfilı), A5, KATO: 19811; Euro.-Sib., Ch.

122. Dorycnium graecum (L.) Ser. -(Ak Kaplanotu), A5, KATO: 19812; Eux., H.

123. Genista januensis Viv. subsp. lydia (Boiss.) Kit Tan \& Ziel. -(Geyik Borcağı), A5, KATO: 19813; E. Medit., Ch.

124. Lathyrus aureus (Stev.) Brandza -(Koru Mürdümüğ̈̈), A5, KATO: 19814; Eux., H. 125. L. laxiflorus (Desf.) O.Kuntze subsp. laxiflorus -(Deli Burçak), A5, KATO: 19815; H.

126. L. pratensis L. -(Y1lan Gürülü), A5, KATO: 19816; Euro.-Sib., H.
127. Medicago falcata L. -(Kart Yonca), A5, KATO: 19817; H.

128. M. lupulina L. -(Bitçikotu), A5, KATO: 19818; Ir.-Tur., Ch.

129. M. orbicularis (L.) Bart. -(Paralık), A5, KATO: 19819; Medit., Th.

130. Melilotus officinalis (L.) Desr. -(Kokulu Yonca), A5, KATO: 19820; Th.

131. Onobrychis oxyodonta Boiss. var. armena (Boiss. \& Huet) Aktoklu -(Kır Korungas1), A5, KATO: 19821; H.

132. Robinia hispida L. -(K1llı Akasya), A5, KATO: 19822; Ph.

133. Trifolium arvense L. var. arvense (Tavşan Ayă̆ı), A5, KATO: 19823; Medit., Th.

134. T. elongatum Willd. -(Helva Üçgülü), A5, KATO: 19824; End., H.

135. T. ochroleucum Huds. -(Mizrak Üçgülü), A5, KATO: 19825; H.

136. T. pratense L. var. pratense -(Çayır Üçgülü), A5, KATO: 19826; H.

137. T. repens L. var. repens -(Ak Üçgül), A5, KATO: 19827; H.

138. Vicia cracca L. subsp. tenuifolia (Roth) Gau. -(Kır Fiği), A5, KATO: 19828; Euro.Sib., H.

139. V. crocea (Desf.) B. Fedtsch. -(Safran Fiği), A5, KATO: 19829; Hyr.-Eux., H.

140. V. freyniana Bornm. -(Delifiğ), A5, KATO: 19830; Eux., End., H.

141. V. sativa L. subsp. sativa -(Fiğ), A5, KATO: 19831; Euro.-Sib., Th.

35. Polygalaceae Hoffmanns. \& Link 142. Polygala major O. F. Mull -(Koca Sütotu), A5, KATO: 19832; Euro.-Sib., H.

143. P. pruinosa Boiss. subsp. pruinosa (Puslu Sütotu), A5, KATO: 19833; Ch.

36. Rosaceae Juss.

144. Agrimonia eupatoria L. subsp. asiatica (Juz.) Skalicky -(Fitıkotu), A5, KATO: 19834; C.

145. Alchemilla caucasica Buser -(Kaf Şebnemlisi), A5, KATO: 19835; Eux. (mt), C.

146. A. mollis (Buser) Rothm. -(Su Keltat1), A5, KATO: 19836; C.

147. Amelanchier ovalis Medik subsp. ovalis -(Kurtağac1), A5, KATO: 19837; E. Medit., $\mathrm{Ph}$.

148. Cerasus avium (L.) Moench -(Kiraz), A5, KATO: 19838; Ph. 
149. Cotoneaster nummularius Fisch. \& C. A. Mey -(Dağ Muşmulası), A5, KATO: 19839; $\mathrm{Ph}$.

150. Crataegus monogyna Jacq. var. monogyna -(Alıç), A5, KATO: 19840; Ph.

151. C. orientalis Pallas ex Bieb. var. orientalis-(Alıç), A5, KATO: 19841; Ph.

152. Filipendula vulgaris Moench (Çayırmelikesi), A5, KATO: 19842; Euro.Sib., $\mathrm{H}$.

153. Fragaria vesca L. -(Dağ Çileği), A5, KATO: 19843 ; $\mathrm{H}$.

154. Geum urbanum L. -(Meryemotu), A5, KATO: 19844; Euro.-Sib., H.

155. Laurocerasus officinalis Roemer (Karayemiş), A5, KATO: 19845; Ph.

156. Malus sylvestris Mill. subsp. orientalis (A. Uglitzkich) Browicz var. orientalis (Yaban Elmas1), A5, KATO: 19846; Ph.

157. Mespilus germanica L. -(Muşmula), A5, KATO: 19847; Eux., Ph.

158. Potentilla recta L. -(Su Parmakotu), A5, KATO: 19848; $\mathrm{H}$.

159. Prunus spinosa L. -(Çakal Eriği), A5, KATO: 19849; Euro.-Sib., Ph.

160. Pyracantha coccinea Roem. (Ateşdikeni), A5, KATO: 19850; Ph.

161. Pyrus communis L. subsp. communis (Bey Armudu), A5, KATO: 19851; Ph.

162. P. elaeagnifolia Pallas subsp. elaeagnifolia -(Ahlat), A5, KATO: 19852; $\mathrm{Ph}$.

163. Rosa boissieri Crep. -(Has Gül), A5, KATO: 19853 ; Ph.

164. $R$. canina L. -(Kuşburnu), A5, KATO: 19854; Ph.

165. Rubus hirtus Waldst. \& Kit. (Ahududu), A5, KATO: 19855; Euro.-Sib., Ch.

166. $R$. idaeus L. -(Tüntürük), A5, KATO: 19856; Ch.

167. Sanguisorba minor L. subsp. balearica (Bourg. ex Nyman) Muñoz Garm. \& C.Navarro -(Kelekayağı), A5, KATO: 19857; H.

168. Sorbus aucuparia L. -(Kuş Üvezi), A5, KATO: 19858; Euro.-Sib., Ph.

169. S. torminalis (L.) Crantz var. torminalis -(Pitlicen), A5, KATO: 19859; Euro.-Sib., $\mathrm{Ph}$.

170. S. umbellata (Desf.) Fritsch -(Geyik Elmas1), A5, KATO: 19860; Ph.

37. Ulmaceae Mirb.
171. Ulmus glabra Hudson -(Dağ Karaağac1), A5, KATO: 19861; Euro.-Sib., $\mathrm{Ph}$.

38. Urticaceae Juss.

172. Urtica dioica L. subsp. dioica -(Isirgan Otu), A5, KATO: 19862; Euro.-Sib., H.

39. Betulaceae Gray

173. Alnus glutinosa (L.) Gaertner-(Adi Kızılağaç), A5, KATO: 19863; Euro.-Sib., $\mathrm{Ph}$

174. Betula pendula Roth -(Salkım Huş), A5, KATO: 19864; Ph.

175. Carpinus betulus L. -(Adi Gürgen), A5, KATO: 19865; Euro.-Sib., Ph.

176. Corylus avellana L. var. avellana -(Adi Find1k), A5, KATO: 19866; Euro.-Sib., Ph.

177. C. colurna L. -(Türk Findığ1), A5, KATO: 20568; Euro.-Sib., Ph.

40. Fagaceae Dumort.

178. Fagus orientalis Lipsky -(Doğu Kay1n1), A5, KATO: 19867; Euro.-Sib., Ph.

179. Quercus infectoria Olivier subsp. veneris (A.Kern) Meikle -(Mazı Meşesi), A5, KATO: 19868; Ph.

180. Q. petraea (Mattuschka) Liebl. subsp. iberica (Steven ex Bieb) Krassiln. -(Sapsiz Meșe), A5, KATO: 19869; Ph.

181. Q. pubescens Willd. subsp. pubescens (Tüylü Meşe), A5, KATO: 19870; Ph.

41. Geraniaceae Juss.

182. Erodium birandianum Ilars. \& Yurdak. -(Paşa İğneliği), A5, KATO: 19871; Eux., End., Ch.

183. E. cicutarium (L.) L'Herit. subsp. cicutarium -(İğnelik), A5, KATO: 19872; Th.

184. Geranium asphodeloides Burm. fil.(Yaramerhemi), A5, KATO: 19873; Euro.Sib., H.

185. G. pyrenaicum Burm. fil. (Gelinçarşafi), A5, KATO: 19874; C.

186. G. robertianum L. -(Dağ Itır1), A5, KATO: 19875; Th.

187. G. tuberosum L. -(Çakmuz), A5, KATO: 19876; C.

42. Lythraceae J. St.-Hil.

188. Lythrum salicaria L. -(Hevhulma), A5, KATO: 19877; Euro.-Sib., H.

43. Onagraceae Juss.

189. Circaea lutetiana L. -(Kankurutan), A5, KATO: 19878; C.

190. Epilobium angustifolium L. -(Yak1otu), A5, KATO: 19879; $\mathrm{H}$. 
191. E. dodonaei Vill. -(Çayırgülü), A5, KATO: 19880; Euro.-Sib., H.

192. E. hirsutum L. -(Hasan Hüseyin Çiçeği), A5, KATO: 19881; H.

193. E. lanceolatum Sebast. \& Mauri (Dilyak1s1), A5, KATO: 19882; H.

194. E. montanum L. -(Dağ Yakısı), A5, KATO: 19883; Euro.-Sib., H.

44. Staphyleaceae Martinov

195. Staphylea pinnata L. -(Ağızlık Çalısı),

A5, KATO: 19884; Ph.

45. Sapindaceae Juss.

196. Acer campestre L.-(Ova Akçaağacı), A5, KATO: 19885; Euro.-Sib., Ph.

197. A. heldreichii Orph. ex Boiss. subsp. trautvetteri (Medw.), A.E.Mur. -(Kafkas Akçaağacı), A5, KATO: 19886; Eux., Ph.

198. A. hyrcanum Fisch. \& Mey. subsp. keckianum (Pax) Yalt. -(Kazdağ Akçaağac1), A5, KATO: 19887; End., Ph.

46. Malvaceae Juss.

199. Malva alcea L. -(Ebecik), A5, KATO: 19888; H.

47. Cistaceae Juss.

200. Cistus laurifolius L. -(Karağan), A5, KATO: 19889; Ph.

201. Helianthemum nummularium (L.) Mill.(Güngülü), A5, KATO: 19890; H.

48. Thymelaeaceae Juss.

202. Daphne oleoides Schreb. subsp. oleoides -(Yabani Defne), A5, KATO: 19891; Ch.

203. D. pontica L. subsp. pontica (Sırımağu), A5, KATO: 19892; Eux., Ph.

49. Brassicaceae Burnett

204. Aubrieta canescens (Boiss.) Bornm., A5, KATO: 19893; End., H.

205. Cardamine bulbifera (L.) Crantz (Dişlikök), A5, KATO: 19894; Euro.-Sib., C. 206. C. impatiens L. var. pectinata (Pallas) Trautv. -(Taraklı Kodimotu), A5, KATO: 19895; Euro.-Sib., C.

207. C. quinquefolia (Bieb.) Schmalh. (Hanımgömleği), A5, KATO: 19896; Euro.Sib., C.

208. Erysimum cuspidatum (Bieb.) DC. (Kuyruklu Zarife), A5, KATO: 19897; H.

209. Hesperis matronalis L. subsp. matronalis -(Akşamyıldızı), A5, KATO: 19898; H.

210. Microthlaspi perfoliatum (L.) F. K. Mey -(Giyle), A5, KATO: 19899; Th.
211. Thlaspi orbiculatum Stev. -(Koru Dağarcığı), A5, KATO: 19900; Th.

50. Resedaceae Martinov

212. Reseda lutea L. var. lutea -(Muhabbet Çiçeği), A5, KATO: 19901; H.

51. Cornaceae Bercht. \& J. Presl.

213. Cornus mas L. -(Kızılc1k), A5, KATO: 19902; Euro.-Sib., Ph.

214. C. sanguinea L. subsp. australis (C. A. Meyer) Jav. -(Kansiğdiren), A5, KATO: 19903; Euro.-Sib., Ph.

52. Ericaceae Juss.

215. Monotropa hypopithys L. (Sarıkuşyuvası), A5, KATO: 19904; C.

216. Pyrola media Sw. -(Meşe Kekliküzüm), A5, KATO: 19905; Euro.-Sib., H.

217. Rhododendron luteum Sweet -( Sar1 Çiçekli Ormangülü), A5, KATO: 19906; Eux., Ph.

218. Vaccinium arctostaphylos L. (Likarpa), A5, KATO: 19907; Eux., Ph.

53. Primulaceae Batsch ex Borkh.

219. Cyclamen coum Mill. var. coum (Yersomunu), A5, KATO: 19908; C.

220. Lysimachia verticiliaris Spreng. -(Hilal Kargaotu), A5, KATO: 19909; Hyr.-Eux., H. 221. Primula acaulis (L.) L. subsp. acaulis (Çuha Çiçeği), A5, KATO: 19910; Euro.Sib., H.

54. Apocynaceae Juss.

222. Vincetoxicum fuscatum Rchb. f. subsp. fuscatum -(Gavur Biberi), A5, KATO: 19911; H.

55. Gentianaceae Juss.

223. Centaurium erythraea Rafn -(Kırmız1 Kantaron), A5, KATO: 19912; Euro.-Sib., H. 224. Gentiana asclepiadea L. -(Sütlü Güşad), A5, KATO: 19913; Euro.-Sib., H.

225. G. septemfida Pallas -(Yedi Gentiyan), A5, KATO: 19914; Hyr.-Eux., H.

56. Rubiaceae Juss.

226. Asperula arvensis L. -(Tarla belumotu), A5, KATO: 19915; Th.

227. A. taurina L. -(Küçük Fevve), A5, KATO: 19916; C.

228. Cruciata taurica (Pallas ex Willd.) Ehr. -(Kırım Güzeli), A5, KATO: 19917; Ir.-Tur., Th.

229. Galium odoratum (L.) Scop. -(Orman İplikçiği), A5, KATO: 19918; Euro.-Sib., H. 230. G. paschale Forsskal -(Gök İplikçik), A5, KATO: 19919; E. Medit., H. 
231. G. rotundifolium L. -(Koru Yopurtotu), A5, KATO: 19920; Euro.-Sib., H.

232. G. verum L. subsp. verum -(Boyalık), A5, KATO: 19921; Euro.-Sib., H.

57. Lamiaceae Martinov

233. Ajuga chamaepitys (L.) Schr. subsp. chia (Schr.) Arcang. -(Acigic1), A5, KATO: 19922; H.

234. A. orientalis L. -(Dağmayası11), A5, KATO: 19923; H.

235. A. reptans L. -(Meryemsaçı), A5, KATO: 19924; Euro.-Sib., H.

236. Clinopodium acinos (L.) Kuntze (Kayrakçayı), A5, KATO: 19925; Euro.-Sib., $\mathrm{H}$.

237. C. grandiflorum (L.) Kuntze -(Kaba Fesleğen), A5, KATO: 19926; Euro.-Sib., H. 238. C. nepeta (L.) Kuntze subsp. glandulosum (Req.) Gov. -(Sümüklü Fesleğen), A5, KATO: 19927; H.

239. C. vulgare $\mathrm{L}$. subsp. arundanum (Boiss.) Nyman -(Yabani Fesleğen), A5, KATO: 19928; Euro.-Sib., H.

240. Lamium album L. subsp. crinitum (Montbret \& Aucher ex Benth.) Mennema (Kovanlık), A5, KATO: 19929; Eux. (mt), H. 241. L. amplexicaule L. var. amplexicaule (Baltutan), A5, KATO: 19930; Euro.-Sib., Th.

242. L. purpureum L. var. purpureum (Ballıbaba), A5, KATO: 19931; Euro.-Sib., Th.

243. Marrubium astracanicum Jacq. subsp. astracanicum -(Mor Yayotu), A5, KATO: 19932; H.

244. M. parviflorum Fisch. \& Mey. subsp. oligodon (Boiss.) Seybold -(Küllü Bozotu), A5, KATO: 19933; $\mathrm{H}$.

245. Mentha longifolia (L.) Hudso subsp. longifolia -(Pünk), A5, KATO: 19934; H.

246. Nepeta nuda L. subsp. albiflora (Boiss.) Gams -(Karaküncü), A5, KATO: 19935; H.

247. Origanum vulgare L. subsp. vulgare (Karakınık), A5, KATO: 19936; H.

248. Prunella laciniata (L.) L.-(Bodur Fesleğen), A5, KATO: 19937; Euro.-Sib., H. 249. P. vulgaris L. -(Gelinciklemeotu), A5, KATO: 19938; Euro.-Sib., H.

250. Salvia aethiopis -(Habeş Adaçayı), A5, KATO: 19939; H.

251. S. cyanescens Boiss. \& Bal. -(Mor Galabor), A5, KATO: 19940; Ir.-Tur., End., $\mathrm{H}$.
252. S. forskahlei L. -(Dolmayaprağı), A5, KATO: 19941; Eux., H.

253. S. glutinosa L. -(Oklu Şalba), A5, KATO: 19942; Hyr.-Eux., H.

254. S. sclarea L. -(Paskulak), A5, KATO: 19943; H.

255. S. tomentosa Miller -(Şalba), A5, KATO: 19944; Medit., Ch.

256. S. verticillata L. subsp. verticillata (Dadırak), A5, KATO: 19945; Euro.-Sib., H.

257. Scutellaria albida L. subsp. velenovskyi (Rech.f.) Greuter \& Burdet -(Benekli Kaside), A5, KATO: 19946; E. Medit., H.

258. S. orientalis L. subsp. pinnatifida J.R.Edm. -(Kırbaç Sırmı), A5, KATO: 19947; H.

259. Sideritis germanicopolitana Bornm. subsp. germanicopolitana -(Karakurbağa Çay1), A5, KATO: 19948; End., H.

260. S. montana L. subsp. remota (D’Urv.) P. W. Ball ex Heywood -(Mor Karaçay), A5, KATO: 19949; Medit., Th.

261. Stachys annua (L.) L. subsp. annua var. апnиа -(Haciosman Otu), A5, KATO: 19950; Th.

262. S. byzantina C. Koch -(Boz Karabaş), A5, KATO: 19951; Euro.-Sib., H.

263. S. setifera C. A. Meyer subsp. lycia (Gand.) Bhattacharfee -(Zarif Deliçay), A5, KATO: 19952; Ir.-Tur., End., H.

264. Teucrium chamaedrys L.-(K1sa Mahmut), A5, KATO: 19953; Euro.-Sib., Ch.

265. T. polium L. subsp. polium -(Ac1 Yavşan), A5, KATO: 19954; H.

266. Thymus sipyleus Boiss. -(Sipil Kekiği), A5, KATO: 19955; Ch.

267. Ziziphora persica Bunge -(Kara Reyhan), A5, KATO: 19956; Ir.-Tur., Th.

58. Orobanchaceae Vent.

268. Euphrasia pectinata Ten. -(Gözotu), A5, KATO: 19957; Euro.-Sib., Th.

269. Macrosyringion glutinosum (M.Bieb.) Rothm. -(Sar1 Gözotu), A5, KATO: 19958; Th.

270. Melampyrum arvense L. var. arvense (İnekbuğday1)，A5，KATO: 19959; Euro.Sib., Th.

271. Orobanche elatior Sutton -(Boylu Canavarotu), A5, KATO: 19960; VP.

272. Pedicularis comosa L. var. sibthorpii (Boi.) Boi. -(Hotozlu Bitotu), A5, KATO: 19961; H. 
273. Rhinanthus angustifolius C.C.Gmelin subsp. grandiflorus (Wallr.) D.A. Webb. (Horozotu), A5, KATO: 19962; Th.

274. Rhynchocorys elephas (L.) Griseb. (Filburnu), A5, KATO: 19963; Euro.-Sib., H.

59. Plantaginaceae Juss.

275. Digitalis ferruginea L. subsp. ferruginea L. -(Arıkovan1), A5, KATO: 19964; Euro.-Sib., H.

276. D. lamarckii Ivanina-(Yüksükotu), A5, KATO: 19965; Ir.-Tur., H.

277. Globularia trichosantha Fisch. \& Mey.(Köse Yayılımı), A5, KATO: 19966; Ir.-Tur., $\mathrm{H}$.

278. Linaria corifolia Desf. -(Tarla Nevruzotu), A5, KATO: 19967; Ir.-Tur., End., $\mathrm{H}$.

279. L. genistifolia (L.) Miller-(Som Nevruzotu), A5, KATO: 19968; Euro.-Sib., $\mathrm{H}$.

280. Plantago lanceolata L. -(Damarlica), A5, KATO: 19969; H.

281. P. major L. subsp. major -(Sinirotu), A5, KATO: 19970; $\mathrm{H}$.

282. Veronica anagallis-aquatica L. (Sugedemesi), A5, KATO: 19971; H.

283. V. chamaedrys L. -(Cancan), A5, KATO: 19972; Euro.-Sib., Ch.

284. V. multifida L. -(Devesabunu), A5, KATO: 19973; Ir.-Tur., End., H.

285. V. serpyllifolia L. -(Güzelnane), A5, KATO: 19974; H.

60. Scrophulariaceae Juss.

286. Scrophularia scopolii Hoppe ex Pers. var. scopolii -(Elköpürten), A5, KATO: 19975; H.

287. Verbascum eriocarpum (Freyn \& Sint.) Bornm. -(Gavur Sığırkuyruğu), A5, KATO: 19976; Eux., H.

288. V. lasianthum Boiss. ex Bentham (Yünlü Sı ğırkuyruğu), A5, KATO: 19977; H. 289. V. pyramidatum Bieb. -(Arsiz Sığırkuyruğu), A5, KATO: 19978; Hyr.Eux., H.

290. V. speciosum Schrader -(Zelve), A5, KATO: 19979; H.

291. V. thapsus L. -(Burunca), A5, KATO: 19980; Euro.-Sib., H.

61. Oleaceae Hoffmanns. \& Link

292. Fraxinus angustifolia Vahl. subsp. angustifolia -(Sivri Dişbudak), A5, KATO: 19981; Ph.
293. Ligustrum vulgare L. -(Kurtbağr1), A5, KATO: 19982; Euro.-Sib., Ph.

62. Convolvulaceae Juss.

294. Convolvulus cantabrica L. (Çadırçiçeği), A5, KATO: 19983; H.

63. Solanaceae Juss.

295. Atropa belladonna L. -(Güzel Avratotu), A5, KATO: 19984; Euro.-Sib., H.

64. Boraginaceae Juss.

296. Anchusa leptophylla Roemer \& Schultes-(Ballık), A5, KATO: 19985; H.

297. Buglossoides arvensis (L.) J.R.Johnst. subsp. sibthorpiana (Griseb.) R. Fern. (Tarla Taşkeseni), A5, KATO: 19986; Th.

298. Cerinthe minor L. subsp. auriculata (Ten.) Domac -(Livarotu), A5, KATO: 19987; H.

299. Cynoglossum officinale L.-(Gözpıtrağı), A5, KATO: 19988; Euro.-Sib., H.

300. Echium vulgare L. subsp. vulgare (Engerek Otu), A5, KATO: 19989; Euro.Sib., H.

301. Myosotis arvensis (L.) Hill(Kardeşboncuğu), A5, KATO: 19990; Euro.Sib., Th.

302. M. lithospermifolia Hornem. -(Taş Boncukotu), A5, KATO: 19991; H.

303. Onosma bornmuelleri Hausskn. (Amasya Şincarı), A5, KATO: 19992; Ir.Tur., End., H.

304. O. isaurica Boiss. \& Heldr. -(Kül Emcek), A5, KATO: 19993; Ir.-Tur., End., $\mathrm{H}$.

305. Trachystemon orientalis (L.) G.Don (Kaldirik), A5, KATO: 19994; Eux., C.

65. Aquifoliaceae Bercht. \& J. Presl.

306. Ilex colchica Poj. -(Çoban Püskülü), A5, KATO: 19995; Eux., Ph.

66. Asteraceae Bercht. \& J. Presl

307. Achillea biserrata Bieb. -(Aksırıkotu), A5, KATO: 19996; Eux., H.

308. A. millefolium L. subsp. millefolium(Civanperçemi), A5, KATO: 19997; Euro.Sib., H.

309. Arctium minus (Hill.) Bernh. -(Löşlek), A5, KATO: 19998; Euro.-Sib., H.

310. Carduus nutans L. subsp. falcatoincurvus P. H. Davis -(Eğri Eşekdikeni), A5, KATO: 19999; H.

311. Centaurea solstitialis L. subsp. solstitialis -(Çakır Dikeni), A5, KATO: 20000; Th. 
312. C. urvillei DC. subsp. stepposa Wagen.-(Yer Kötürümü), A5, KATO: 20001; Ir.-Tur., H.

313. Cichorium intybus L. -(Hindiba), A5, KATO: 20002; H.

314. Cirsium arvense (L.) Scop. (Köygöçüren), A5, KATO: 20003; H.

315. C. hypoleucum DC. -(Vişne Kangali), A5, KATO: 20004; Eux., H.

316. C. vulgare (Savi) Ten. -(Yaygın Kangal), A5, KATO: 20005; H.

317. Cyanus reuterianus (Boiss.) Holub var. phrygia Boram.-(Kapele), A5, KATO: 20006; E. Medit., End., H.

318. C. triumfettii (All.) Dostál ex Á.Löve \& D.Löve-(Deli Kapele), A5, KATO: 20007; H.

319. Doronicum orientale Hoffm. (Kaplanotu), A5, KATO: 20008; H.

320. Echinops microcephalus SM. (Papazkalpağ1), A5, KATO: 20009; Medit., $\mathrm{H}$.

321. Erigeron acris L. subsp. pycnotrichus (Vierh.) Grierson -(Yünlü Şifaotu), A5, KATO: 20010; Euro.-Sib., Th.

322. Helichrysum arenarium (L.) Moench subsp. aucheri (Boiss.) Davis \& Kupicha (Yayla Çiçeği), A5, KATO: 20011; Ir.-Tur., End., $\mathrm{H}$.

323. Hieracium pannosum Boiss. (Acıkanak), A5, KATO: 20012; E. Medit. (mt), H.

324. Inula oculus-christi L. -(Yol Otu), A5, KATO: 20013; Euro.-Sib., H.

325. Jurinea consanguinea DC. (Geyikgöbeği), A5, KATO: 20014; H.

326. Lactuca muralis (L.) Gaertn. -(Divar Marulu), A5, KATO: 20015; Euro.-Sib., Th.

327. L. serriola L. -(Eşekhelvası), A5, KATO: 20016; Euro.-Sib., H.

328. Lapsana communis L. subsp. intermedia (Bieb.) Hayek-(Şebrek), A5, KATO: 20017; $\mathrm{H}$.

329. Leontodon asperrimus (Willd.) Ball (Aşyemliği), A5, KATO: 20018; Ir.-Tur., H.

330. L. hispidus L. subsp. hispidus (Gulikazer), A5, KATO: 20019; Euro.-Sib., $\mathrm{H}$.

331. Petasites hybridus (L.) Gaer, Me. \& Schb. -(Kabalak), A5, KATO: 20020; Euro.Sib., C.

332. Pilosella hoppeana (Schultes) C. H. \& F. W. Schultz subsp. testimonialis (Naegli ex
Peter) P.D.Sell \& C.West -(Saplı Tirnakotu), A5, KATO: 20021; $\mathrm{H}$.

333. P. hoppeana (Schultes) C. H. \& F. W. Shultz subsp. troika (Zahn) Sell \& West -(Er Tirnakotu), A5, KATO: 20022; H.

334. Pulicaria dysenterica subsp. dysenterica (L.) Bernh. -(Yaraotu), A5, KATO: 20023; $\mathrm{H}$.

335. Reichardia dichotoma (Vahl) Freyn (Karasak1z), A5, KATO: 20024; Ir.-Tur., H. 336. Senecio pseudo-orientalis Schischkin (Sar1 Şiro), A5, KATO: 20025; Ir.-Tur., H. 337. S. vernalis Waldst. \& Kit. (Kanaryaotu), A5, KATO: 20026; Th.

338. Solidago virgaurea L. -(Altınbaşak Çiçeği), A5, KATO: 20027; Euro.-Sib., H.

339. Sonchus asper (L.) Hill subsp. glaucescens (Jord.) Ball. -(Gevirtlek), A5, KATO: 20028; $\mathrm{H}$.

340. Tanacetum poteriifolium (Ledeb.) Grierson -(Dişlek Pireotu), A5, KATO: 20029; Eux., H.

341. Taraxacum buttleri Soest (Karahindiba), A5, KATO: 20030; H.

342. Telekia speciosa (Schreber) Baumg. (Puğre), A5, KATO: 20031; Euro.-Sib., H. 343. Tephroseris integrifolia (L.) Holub subsp. aucheri (DC.) B.Nord. -(Ümbülük Çiçeği), A5, KATO: 20032; Euro.-Sib., H.

344. Tragopogon dshimilensis K. Koch (Cimil Porini), A5, KATO: 20033; Euro.Sib., End., H.

345. T. coloratus C. A. Meyer -(Katır Yemliği), A5, KATO: 20034; Ir.-Tur., H.

346. T. porrifolius L. subsp. abbreviates (Boiss.) Coşkunçelebi \& M. Gültepe (Çayır Yemliği), A5, KATO: 20035; H.

347. T. pratensis L. -(Salsifin) A5, KATO: 20036; Euro.-Sib., H.

348. Tripleurospermum tenuifolium (Kit.) Freyn -(Saçaklı Beybunik), A5, KATO: 20037; Euro.-Sib., H.

349. Turanecio hypochionaeus (Boiss.) Hamzaoğlu -(Turanotu), A5, KATO: 20038; $\mathrm{H}$.

350. Tussilago farfara L. -(Öksürükotu), A5, KATO: 20039; Euro.-Sib., C.

351. Xeranthemum cylindraceum SM. -(Deli Kağıtçiçeği), A5, KATO: 20040; Th.

67. Campanulaceae Juss.

352. Asyneuma amplexicaule (Willd.) Hand.Mazz -(Hoşdeğnek), A5, KATO: 20041; H. 
353. A. limonifolium (L.) Janch. subsp. pestalozzae (Boiss.) Damboldt (Tavşanekmeği), A5, KATO: 20042; End., $\mathrm{H}$.

354. A. rigidum (Willd.) Grossh. subsp. rigidum -(Nujdan), A5, KATO: 20043; Ir.Tur., $\mathrm{H}$.

355. Campanula glomerata L. subsp. hispida (Witasek) Hayek -(Yumak Çanı), A5, KATO: 20044; Euro.-Sib., H.

356. C. latiflora M.Bieb. subsp. latifolia (Çançiçeği), A5, KATO: 20045; Euro.-Sib., $\mathrm{H}$.

357. C. olympica Boiss. -(Orman Çan1), A5, KATO: 20046; Eux., H.

358. C. rapunculus L. var. lambertiana (A. DC.) Boiss. -(Sidikli Çançiçeği), A5, KATO: 20047; Euro.-Sib., H.

68. Apiaceae Lindl.

359. Chaerophyllum aureum L. (Sarlakotu), A5, KATO: 20048; H.

360. C. byzantium Boiss. -(H1lakotu), A5, KATO: 20049; Eux., H.

361. Heracleum platytaenium Boiss. (Öğrekotu), A5, KATO: 20050; Eux., H.

362. Laser trilobum (L.) Borkh -(Kefe Kimyonu), A5, KATO: 20051; H.

363. Pastinaca sativa L. subsp. urens (Req. Ex Godron) Celak -(Şeker Havucu), A5, KATO: 20052; H.

364. Sanicula europaea L. -(Sanikel), A5, KATO: 20053; Medit., H.

365. Torilis arvensis (Huds.) Link subsp. arvensis -(Dercik Otu), A5, KATO: 20054; Th.

69. Araliaceae Juss.

366. Hedera helix L. f. helix -(Duvar sarmaşığı), A5, KATO: 20055; Ph.

70. Adoxaceae E. Mey. (Mürvergiller)

367. Sambucus ebulus L. -(Mürver Otu), A5, KATO: 20056; Euro.-Sib., H.

368. S. nigra L. -(Ağaç Mürver), A5, KATO: 20057; Euro.-Sib., Ph.

369. Viburnum lantana L. -(Germeşe), A5, KATO: 20058; Euro.-Sib., Ph.

71. Caprifoliaceae Juss.

370. Cephalaria syriaca (L.) Schrader (Pelemir), A5, KATO: 20059; H.

371. Dipsacus laciniatus L. -(Fesçitarağı), A5, KATO: 20060; $\mathrm{H}$.

372. Knautia involucrata Somm. \& Lev. (Deli Eşekkulağı), A5, KATO: 20061; Eux. (mt), H.
373. Lonicera orientalis Lam. -(Has Çakkana), A5, KATO: 20062; End., Ph.

374. Valeriana alliarifolia Adams -(Pisot), A5, KATO: 20063; $\mathrm{H}$.

\section{Abbreviations}

The abbreviations used in the text and the floristic list are as follows: Eux.: Euxine, Eur.-Sib.: Euro-Siberian, Hyr.-Eux.: Hyrcano-Euxine, Eux. (mt): Euxine (mountain), Medit.: Mediterranean, E. Medit.: East Mediterranean, E. Medit. (mt): East Mediterranean (mountain), Ir.-Tur.: Irano-Turanian, IUCN: International Union for the Conservation of Nature and Natural Resources, End.: Endemic, EN: Endangered, VU: Vulnerable, NT: Near Threatened, LC: Least Concern, Ph.: Phanerophyte, H.: Hemicryptophyte, C.: Cryptophyte, Ch.: Chamaephyte, Th.: Therophyte, VP.: Vascular Parasite, KATO: Herbarium of the Faculty of Forestry, Karadeniz Technical University, No.: Number.

\section{Acknowledgement}

The authors want to express their special thanks to staff of Kastamonu Regional Directorate for their logistic help supplying plant material.

\section{References}

Aklıbaşında, M., Bulut, Y. \& Külekçi, E. A. (2012). Aladağlar'da (Kayseri-Yahyalı) Yetişen Doğal Bitkilerin Flora Turizmi Açısından Değerlendirilmesi, KSÜ, Doğa Bilimleri Dergisi, Özel Sayı, 8-15.

Anonymous (2018). Botanic tourism. http://www.kulturturizm.gov.tr/EN99217/activities.html

Avc1, M. (2005). Diversity and endemism in Turkey's vegetation, Coğrafya Dergisi, 13, 27-55.

Baysal, M. (2008). The vascular plants of the Çangal forests (Sinop- Ayancik), Phd, A.U., Graduate School of Natural and Applied Sciences, Ankara.

Bonnier, G. (1912-1934). Flora Compléte Illustrée en Couleurs de France Suisse et Belgique, Neuchatel, Paris, Bruxelles, v. 1-5.

Cain, S.A. (1950). Life-forms and phytoclimate. The Botanical Review, 16(1), 1-32.

Christenhusz, M.J.M. \& Byng, J.W. (2016). The number of known plants species in the world and its annual increase. Phytotaxa, 261(3), 201-217. 
Christenhusz, M.J.M., Zhang, X.C., Schneider, H. (2011a). A linear sequence of extant families and genera of lycophytes and fern, Phytotaxa, 19, 7-54.

Christenhusz, M.J.M., Reveal, J.L., Farjon, A. Gardner, M.F., Mill, R.R. \& Chase, M.W. (2011b.) A new classification and linear sequence of extant Gymnosperms, Phytotaxa, 19, 55-70.

Davis, P.H., Harper, P.C. \& Hedge, I.C. (1971). Plant Life of South-West Asia, The Botanical Society of Edinburgh.

Davis, P.H. (1965-1985). Flora of Turkey and the East Aegean Islands, Vol. I-IX, University Press, Edinburgh.

Davis, P.H., Mill, R.R. \& Tan, K. (1988). Flora of Turkey and the East Aegean Islands, Vol. X, Supplement, University Press, Edinburgh.

Ekim, T. (2002). Türsab Botanik Semineri Notları. Türsab Ar-Ge Departmanı.

Eminağaoğlu, Ö. \& Anşin, R. (2004). Flora of the Karagöl-Sahara National Park (Artvin) and Its Environs. Turkish Journal of Botany, 28(6), 557-590.

Eminağaoğlu, Ö. \& Akyıldırım B., H. (2015). Artvin'in Genel Tanıtımı, s: 1-24. Şu eserde: Eminağaoğlu, Ö. (Ed.), Artvin'in Doğal Bitkileri, İstanbul: Promat.

Güner, A., Özhatay, N., Ekim, T. \& Başer, K.H.C. (2000). Flora of Turkey and the East Aegean Islands and Suppl. Vol. XI, Edinburgh Univ. Press.

Güner, A., Aslan, S., Ekim, T., Vural, M. \& Babaç, M.T. (eds.). (2012). Turkish Plant List (Vascular Plants), NGBB ve Flora Araştırmaları Derneği Yayını, İstanbul.

Irmak, M. A. \& Yilmaz, H. (2011). Determination of perception of flora tourism via questionnaire surveys. Biological Diversity and Conservation, 4(1), 99-106.

Kanoğlu, E. (2002). Flora of Abana (Kastamonu) and its environments, Master's thesis, K.T.U., Graduate School of Natural and Applied Sciences, Trabzon.

Karaburç, İ. (2006). Oyrak pass and its environment's flora, Master's thesis, G.Ü., Graduate School of Natural and Applied Sciences, Ankara.

Karaköse, M. (2015). Flora, vegetation and classification of habitats of Yaraligöz education and observation forest (Kastamonu) and Finike forest planning unit (Antalya), Phd thesis, K.T.U., Graduate School of Natural and Applied Sciences, Trabzon.

Ketenoğlu, O. \& Güney, K. (1997). Batı Küre Dağları (Kastamonu-İnebolu-Cide) Florasına Katkılar, Ot Sistematik Botanik Dergisi, 4, 3960 .
Kılınç, M. (1985a). İç Anadolu Batı Karadeniz Geçiş Bölgesi'nde Devrez Çayı ile Kızılırmak Arasında Kalan Bölgenin Florası, Doğa Bilim Dergisi, A2, 9(2), 283-314.

Kılınç, M. (1985b). İç Anadolu-Batı Karadeniz Geçiş Bölgesi'nde Devrez Çayı ile Kızılırmak Arasında Kalan Bölgenin Vejetasyonu, Doğa Bilim Dergisi, A2, 9(2), 315-357.

Korkmaz, H. \& Engin, A. (2001). The Flora of the Boyabat (Sinop) Dam and Its Environs, Turkish Journal of Botany, 25, 397-435.

Kurt, L. (1992). A Phytosociological study on the vegetation of the Köklüce mountain, Master's thesis, A.U., Graduate School of Natural and Applied Sciences, Ankara.

Kurdoğlu, O, Akbulut, S. (2015). Türkiye'de Acil ve Öncelikle Korunması Gereken Bir Alan: Kamilet ve Durguna Vadileri (Arhavi) ve Koruma Gerekçeleri. Kastamonu Üniversitesi Orman Fakültesi Dergisi, 15 (2): 279-296.

Kurdoğlu, O. (2002). Investigation of Kaçkar Mountains National Park and its near environs from the natural resources management point of view, Phd thesis, K.T.U., Graduate School of Natural and Applied Sciences, Trabzon.

Naqinezhad, A., Zare-Maivan, H. \& Gholizadeh, H. (2015). A floristic survey of the Hyrcanian forests in Northern Iran, using two lowlandmountain transects. Journal of forestry research, 26(1), 187-199.

Özbek, M.U. (2004). Flora of Kurtgirmez Mountain and Çatak Canyon (Küre Mountains-Kastamonu), Master's thesis, G.Ü., Graduate School of Natural and Applied Sciences, Ankara.

Özcan, G.E. (2017). The Impacts of Ips sexdentatus on the Moisture Content of Anatolian Black Pine Trees. Kastamonu Üniversitesi Orman Fakültesi Dergisi, 17 (1): 99-106.

Özen, F. \& Kılınç, M. (1995). Flora of regions between Alaçam-Gerze and BoyabatDurağan, Turkish Journal of Botany, 19, 241275.

Özen, M.D., Özbek, M.U. \& Vural. M. (2013). Flora of Armutluçayır (Kastamonu/Turkey), Biological Diversity and Conservation, 6(1), 22-31.

Özhatay, N., Kültür, Ş. \& Gürdal, B. (2013). Check-list of additional taxa to the Supplement Flora of Turkey VI. Journal of Faculty of Pharmacy of Istanbul University, 43(1), 33-82.

Özhatay, N., Kültür, Ş. \& Gürdal, B. (2015). Check-list of additional taxa to the Supplement Flora of Turkey VII. Journal of Faculty of Pharmacy of Istanbul University, 45(1), 61-86. 
Özhatay, N., Kültür, Ş. \& Gürdal, B. (2017). Check-list of additional taxa to the supplement flora of Turkey VIII. Journal of Faculty of Pharmacy of Istanbul University, 47(1), 30-44.

Palabaş Uzun, S. \& Anşin, R. (2006). Subalpine and alpine flora of Altındere valley (Maçka, Trabzon). Turkish Journal of Botany, 30(5), 381-398.

Philips, R. (1994). Grasses, Ferns, Mosses \& Lichens of Great Britain and Ireland, Macmillan Publishers Ltd., 306.

Raunkiaer, C. (1934). The life forms of plants and statictical plant geography, Clarendon Press, Oxford, 147.

Stevens, P.F. (2001). Angiosperm Phylogeny Website, Version 12, (and more or less continuously updated since). http://www.mobot.org/MOBOT/research/AP web/. 10 Ekim 2012.

Şen, G, Buğday, S.E. (2015). Kastamonu İlinde çeşitli statülerde koruma ve kullanma amaçlı belirlenmiş alanlar. Kastamonu Üniversitesi Orman Fakültesi Dergisi, 15(2): 214-230.

Tekin, E. (2007a). Türkiye'nin En Güzel Yaban

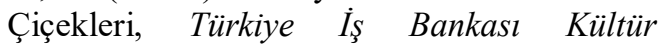
Yayınlarl, 1, 638.

Tekin, E. (2007b). Türkiye'nin En Güzel Yaban Çiçekleri, Türkiye $\dot{I}_{\text {ş Bankası Kültür }}$ Yayınları, 2, 420.

Terzioğlu, S., Bilgili, E., \& Karaköse, M. (2009). Forest of Turkey, OGM, Eğitim Dairesi Başkanlığı, Ankara.

Trauer, B. (2006). Conceptualizing special interest tourism-frameworks for analysis. Tourism management, 27(2), 183-200.

Uzun, A. \& Terzioğlu, S. (2008). Vascular flora of forest vegetation in Altındere valley (Maçka-Trabzon). Turkish Journal of Botany, 32(2), 135-153.

Yaltırık, F. (1967). Acer L. In: Davis PH (ed) Flora of Turkey and the East Aegean Islands, Edinburgh University Press, Edinburgh, 2, 509-519.

Yaman, K. \& Akyıldı, M. H. (2008). The costs of collecting, processing and marketing of some non-wood forest products in Kastamonu. Kastamonu University Journal of Forestry Faculty, 8(1), 26-36.

Yurdakulol, E., Demirörs, M. \& Yıldı, A. (2002). A phytosociological study of the vegetation of the Devrekani-Inebolu-Abana area (Kastamonu-Turkey), Issrail Journal of Science, 50, 293-311. 